ARTICLE

\title{
Engineering of atomic-scale flexoelectricity at grain boundaries
}

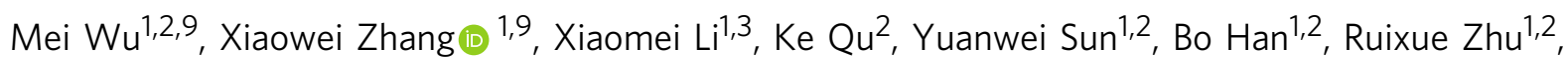

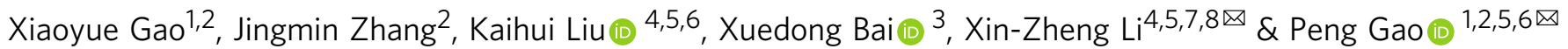

Flexoelectricity is a type of ubiquitous and prominent electromechanical coupling, pertaining to the electrical polarization response to mechanical strain gradients that is not restricted by the symmetry of materials. However, large elastic deformation is usually difficult to achieve in most solids, and the strain gradient at minuscule is challenging to control. Here, we exploit the exotic structural inhomogeneity of grain boundary to achieve a huge strain gradient $\left(\sim 1.2 \mathrm{~nm}^{-1}\right)$ within 3-4-unit cells, and thus obtain atomic-scale flexoelectric polarization of up to $\sim 38 \mu \mathrm{C} \mathrm{cm}^{-2}$ at a $24^{\circ} \mathrm{LaAlO}_{3}$ grain boundary. Accompanied by the generation of the nanoscale flexoelectricity, the electronic structures of grain boundaries also become different. Hence, the flexoelectric effect at grain boundaries is essential to understand the electrical activities of oxide ceramics. We further demonstrate that for different materials, altering the misorientation angles of grain boundaries enables tunable strain gradients at the atomic scale. The engineering of grain boundaries thus provides a general and feasible pathway to achieve tunable flexoelectricity.

\footnotetext{
${ }^{1}$ International Center for Quantum Materials, School of Physics, Peking University, Beijing 100871, China. ${ }^{2}$ Electron Microscopy Laboratory, School of Physics, Peking University, Beijing 100871, China. ${ }^{3}$ Beijing National Laboratory for Condensed Matter Physics and Institute of Physics, Chinese Academy of Sciences, Beijing 100190, China. ${ }^{4}$ State Key Laboratory for Mesoscopic Physics, School of Physics, Peking University, Beijing 100871, China. ${ }^{5}$ Collaborative Innovation Centre of Quantum Matter, Beijing 100871, China. ${ }^{6}$ Interdisciplinary Institute of Light-Element Quantum Materials and Research Center for Light-Element Advanced Materials, Peking University, Beijing 100871, China. ${ }^{7}$ Frontiers Science Center for Nano-optoelectronics, School of Physics, Peking University, Beijing 100871, China. ${ }^{8}$ Peking University Yangtze Delta Institute of Optoelectronics, Nantong 226010 Jiangsu, China. ${ }^{9}$ These authors contributed equally:

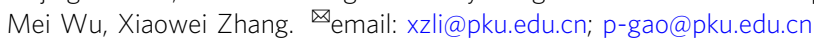


F lexoelectricity is an emergent phenomenon that describes electric polarization controlled by inhomogeneous deformation, i.e., strain gradients, with broken inversion symmetry ${ }^{1,2}$. The flexoelectric effect has two basic features. One is its inherent universality, which allows the appearance of net polarization from strain gradients for every dielectric material, breaking the limit of piezoelectricity that merely exists in the piezoelectric materials of 20 noncentrosymmetric groups ${ }^{1,3}$. Second, when a given strain field is imposed, strain gradients and hence flexoelectricity are expected to be more considerable with the reduction of the spatial scale ${ }^{4,5}$. Therefore, the flexoelectric effect is pronounced at the nanoscale $e^{6-9}$ and has the potential to mediate local polar switching ${ }^{10,11}$, piezoelectric performance ${ }^{12,13}$, optoelectronic characteristics ${ }^{14}$, magnetic properties ${ }^{15,16}$, etc. In particular, dielectric oxides have a large flexoelectric coefficient and thus hold broad promise for application in future nanosized devices ${ }^{11,17}$. However, bending these brittle ceramic materials to realize appropriate and controllable strain gradients is difficult, hindering the achievement of a high flexoelectric response.

Grain boundaries (GBs), which consist of unique periodic arrangements of structural units, can exhibit different properties that are not present in intrinsic bulk crystals ${ }^{18-22}$. For a wellbonded symmetric GB, the atomic configuration of the GB core is a natural trapezoidal shape with a significantly inhomogeneous strain distribution in space. Such disrupted atomic bonding is usually confined to a few unit cells around the GB core, which is expected to introduce huge strain gradients ${ }^{23}$. In addition, tailoring the trapezoidal shape simply by controlling the orientations of two adjacent grains is predictable and practicable, resulting in an adjustable strain gradient as well as flexoelectric polarization. As a result, GBs may serve as a feasible component to realize remarkable and tunable nanoscale flexoelectricity. However, in practice, validating this speculation is challenging, as GBs are of atomic size and have complex structures, while conventional macroscopic characterizations and measurements only provide collective information about the bulk material and may be influenced by other irrelevant factors, such as surface effects $^{24,25}$.

Here, we verify this speculation and develop a general strategy to generate atomic-scale flexoelectric polarization via GB engineering. For a $24^{\circ}$ tilt $\mathrm{LaAlO}_{3}(\mathrm{LAO}) \mathrm{GB}$, we directly visualize the atomic arrangements by advanced atomically resolved scanning transmission electron microscopy (STEM) and spectroscopy techniques and find a huge strain gradient $\left(\sim 1.2 \mathrm{~nm}^{-1}\right)$ and a remarkable flexoelectric effect that induces atomic displacement up to $81.4 \pm 10.5 \mathrm{pm}$ within 3-4-unit cells around the GB. In such a confined GB region, a large local polarization $\left(\sim 38 \mu \mathrm{C} \mathrm{cm}^{-2}\right.$, estimated by first-principles density functional theory (DFT) calculations) exists, forming a "head-to-head" polarization configuration. Electron energy loss spectroscopy (EELS) illustrates that the flexoelectric phase at the GB has stronger hybridization of $\mathrm{La}-\mathrm{O}$ interactions. The presence of nanoscale flexoelectricity accompanied by a change in the electronic structures is expected to play a considerable role in the transport properties of electroceramics. Furthermore, when extrapolated to $\mathrm{SrTiO}_{3}(\mathrm{STO})$ GBs, huge strain gradients also exist in the $22.6^{\circ} \mathrm{GB}$ core, and even larger strain gradients are observed in the $36.8^{\circ} \mathrm{GB}$, exhibiting that tunable flexoelectricity can be achieved by altering the misorientation angle of GBs in different materials. The demonstrated generality of atomic-scale flexoelectricity at GBs provides essential knowledge to understand the electrical activity of oxide ceramics. The tunability via GB engineering may open vistas in nanoelectronics and nanoelectromechanical systems.

\section{Results}

GB engineering design. Figure 1a shows the distinctive geometry of the GB in which huge strain gradients are achievable. Atoms in a symmetric tilt GB are confined by the perfect bulk matrix, subsequently forming periodic arrangements of trapezoidal structural units with large structural deformation, as highlighted in Fig. 1a. Generally, considerable flexoelectricity can only be obtained from significantly bent crystals ${ }^{2}$. In contrast, such an ideal atomic-sized trapezoid in the GB core is expected to generate a huge strain gradient and introduce a large dipole moment within a single unit cell. Based on the geometric relationship of the trapezoid-shaped unit (see "Methods" for details), we obtain the strain gradients in the GB core for different misorientation angles of the GB (Fig. 1b).

Atomic-scale GB structure. Now, we verify the possible presence of flexoelectricity in such a structure by investigating a $24^{\circ}$ tilt LAO GB. LAO has no known ferroelectric instability ${ }^{26}$ and is an insulating oxide typically used as a gate dielectric. The LAO bicrystal was fabricated by thermal diffusion bonding 27,28 , the correlative experimental details are presented in Methods. To pinpoint the atomic configurations and chemistries of the inhomogeneity, we employed various advanced STEM techniques. Figure 2a depicts the high-angle annular dark-field (HAADF) a
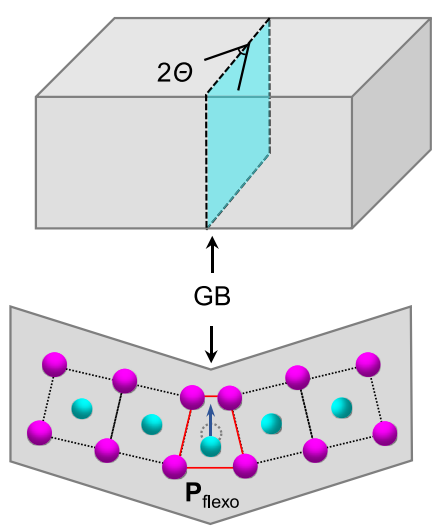

b

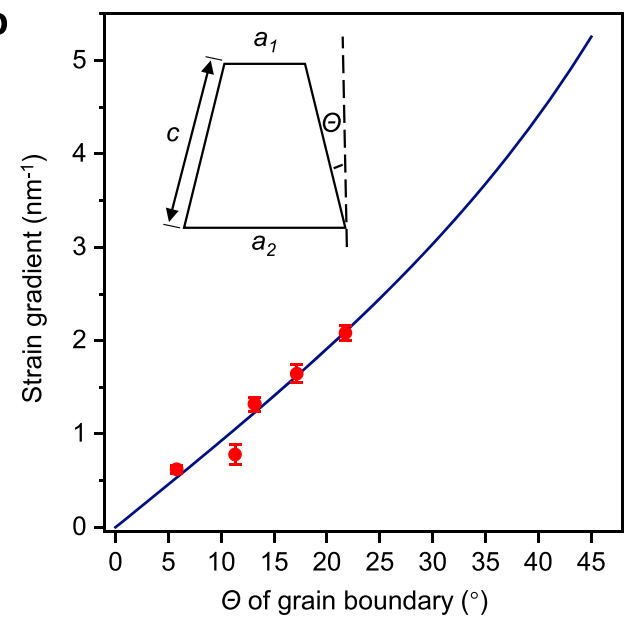

Fig. 1 Schematics of the design of a huge strain gradient via GB engineering. a Schematic of inhomogeneous deformation of the unit cell in a GB (flexoelectric coefficient $f_{11} \neq 0$ ), producing a net dipole moment due to flexoelectricity $\left(\mathbf{P}_{\text {flexo }}\right.$ ). $\mathbf{b}$ Expected distribution of the strain gradient for different GB angles. The red dots represent experimental data from LAO and STO GBs. The error bar is the standard derivation. 
a

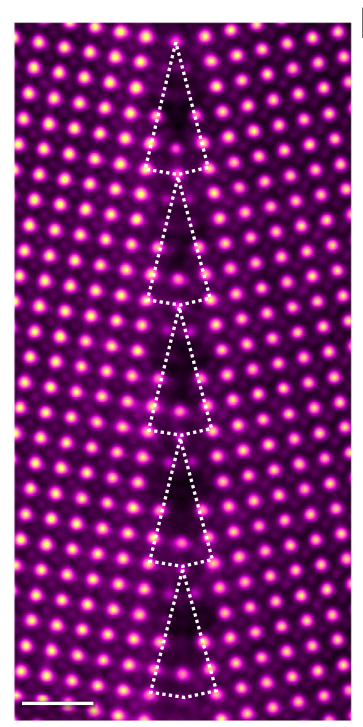

b

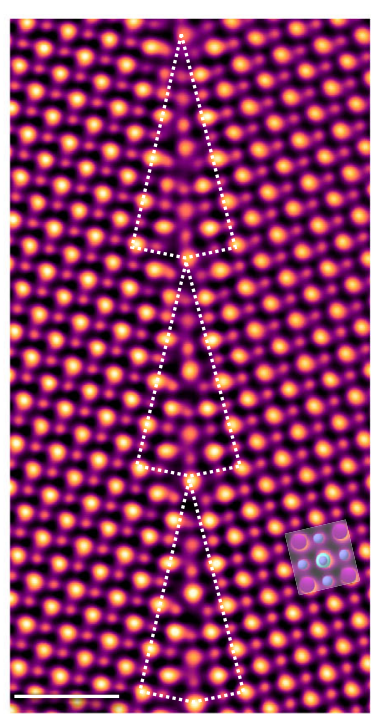

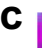

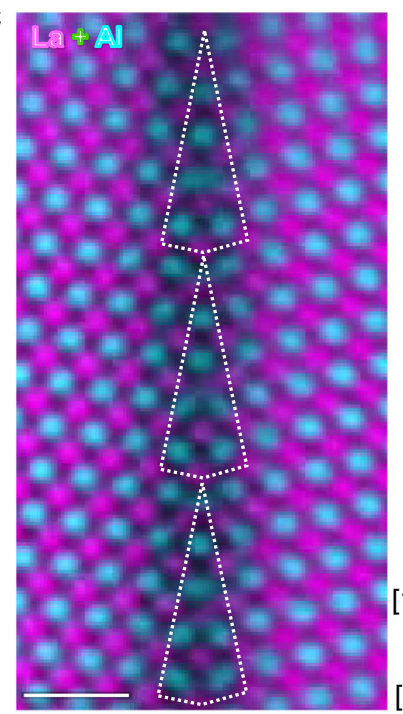

d

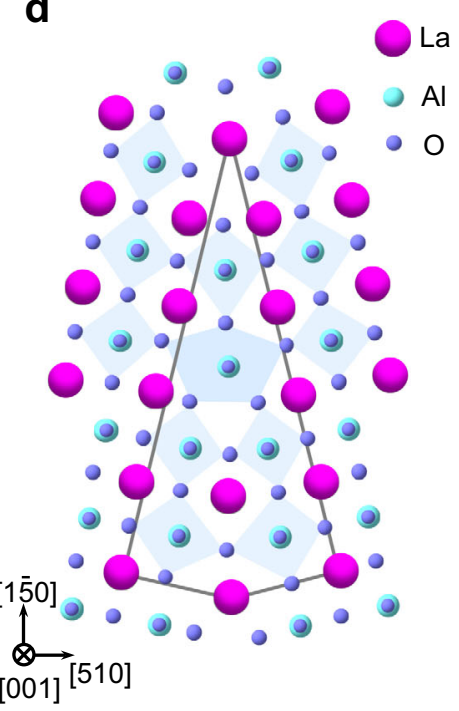

Fig. 2 Atomic arrangements of the LAO GB. a HAADF image. The periodic structural units are highlighted by the white polygons. Scale bar, $1 \mathrm{~nm}$. $\mathbf{b}$ iDPC image depicting the oxygen and cationic arrangement. Scale bar, $1 \mathrm{~nm}$. c Atomically resolved EDS mapping to determine the cationic configuration in the GB. Magenta: La; cyan: Al. Scale bar, $1 \mathrm{~nm}$. d Schematic representation of the complete atomic structure of the LAO GB illustrating structural distortion at the GB. Magenta: La; cyan: Al; purple: O.

image (colored for clarity) viewed along the [001] direction, where structural units are periodically distributed along the [150] projection highlighted by the dotted polygons. The brighter atom columns correspond to La columns, and the other atom type is $\mathrm{Al}$, while the $\mathrm{O}$ columns are invisible due to the $\mathrm{Z}$-contrast sensitivity of HAADF imaging. We, therefore, acquired an integrated differential phase contrast (iDPC) image (Fig. 2b), in which both cations and oxygen atoms are directly visible ${ }^{29}$. Combined with atomically resolved energy dispersive X-ray spectroscopy (EDS), which can be used to further check the possible chemical mixing in the GB (Fig. 2c), we ultimately determined the atomic arrangement in the buried GB, as schematically shown in Fig. 2d. Quantitative elemental analysis is also shown in Supplementary Fig. 1 to reveal the local chemistry across the GB, experimentally showing no distinguishable chemical intermixing or segregation. From the iDPC image, the oxygen octahedron distortions are recognizable at a few angstroms wide around the GB. The trapezoid-shaped units in the core structure of the GB impose huge strain gradients and lead to substantial distortion and shifts of $\mathrm{AlO}$ columns, intuitively visible to the naked eye.

Quantitative analysis of nanoscale flexoelectricity. To better characterize the flexoelectric effect at the GB, a map of atomic displacements was extracted based on the iDPC image, as illustrated in Fig. 3a, wherein the overlaid vectors represent the magnitude and direction of the AlO column offsets with respect to the center of the surrounding four La columns. Such a cationic displacement measurement can minimize the tilt effect ${ }^{30}$, which is inevitable for GB measurement, as the two grains are not exactly identical in terms of zone axes (see details in the Methods and Supplementary Fig. 2). A "head-to-head" configuration pattern of polar vectors emerges around the GB. Note that the magnitude of these vectors is typically higher than the level of noise and possible artifacts (see details in the "Methods" section). To further evaluate the flexoelectric performance of the GB, we quantitatively mapped the strain and strain gradient based on the cation sublattices in the iDPC image (Fig. $3 \mathrm{~b}-\mathrm{e}$ ). The surrounding grains away from the GB were regarded as the reference of a perfect (unstrained) crystal. The strength of the strain parallel to the GB plane $\left(e_{x x}\right)$ is small (Fig. $3 \mathrm{c}$ ), while the strain gradients from $e_{z z}$ along the horizontal $\left(\mathrm{d}\left(e_{z z}\right) / \mathrm{d} z\right.$ in Fig. $\left.3 \mathrm{~d}\right)$ and vertical $\left(\mathrm{d}\left(e_{z z}\right) / \mathrm{d} x\right.$ in Fig. 3e) directions are noticeable within 1 unit cell away from the structural unit. Such a highly nonuniform strain distribution around the GB core is also verified by the geometric phase analysis (GPA) in Supplementary Fig. $3^{31}$. In particular, the unique core structure of the GB (marked by the red trapezoid in Figs. 3a and $4 \mathrm{a}$ ) undergoes drastic fluctuation of the strain gradient up to $1.2 \mathrm{~nm}^{-1}\left(\mathrm{~d}\left(e_{z z}\right) / \mathrm{d} x\right)$, producing a significant polar displacement $(\sim 81.4 \pm 10.5 \mathrm{pm})$ which is expected to generate large flexoelectric polarization (more details on error estimation are included in the "Methods" section), while the strain gradients $\mathrm{d}\left(e_{z z}\right) / \mathrm{d} z$ are negligible due to the symmetric geometry along this direction. For the shear strain, its contribution to the overall flexoelectricity should be very small according to previous theoretical studies ${ }^{32}$.

Based on the DFT calculation in Fig. 4a, the maximum polarization of this trapezoidal-shaped unit is evaluated as $\sim 38$ $\mu \mathrm{C} \mathrm{cm}^{-2}$. The longitudinal flexoelectric coefficient $f_{11}$ of LAO can then be estimated as $0.3 \mathrm{nC} \mathrm{m}^{-1}$, manifesting reasonable agreement with the values in a previous work obtained from theoretical calculations of similar materials ${ }^{25}$. Despite the small difference due to the complex environment of the GB core, the overall atomic structure in the GB presented by DFT calculations is in decent agreement with the experimental results, as illustrated in Supplementary Fig. 4. Hence, the simple consideration of the off-centering between cationic columns delivers the main features of the polar vector distribution, except for the red polyhedron in Figs. $3 \mathrm{a}$ and $4 \mathrm{a}$, in which the calculation shows that the displacement between the cations and oxygen atoms in the AlO octahedron has an upward component and dominates the polar vector, causing a discrepancy with the experimental cationic displacement results. Interestingly, the polarizations around the GB also point toward the GB core, forming a "head-to-head" configuration with an average value of $\sim 10 \mu \mathrm{C} \mathrm{cm}^{-2}$ from each grain side, analogous to the charged domain wall in ferroelectrics. Furthermore, the charge density distribution calculated by DFT 
a

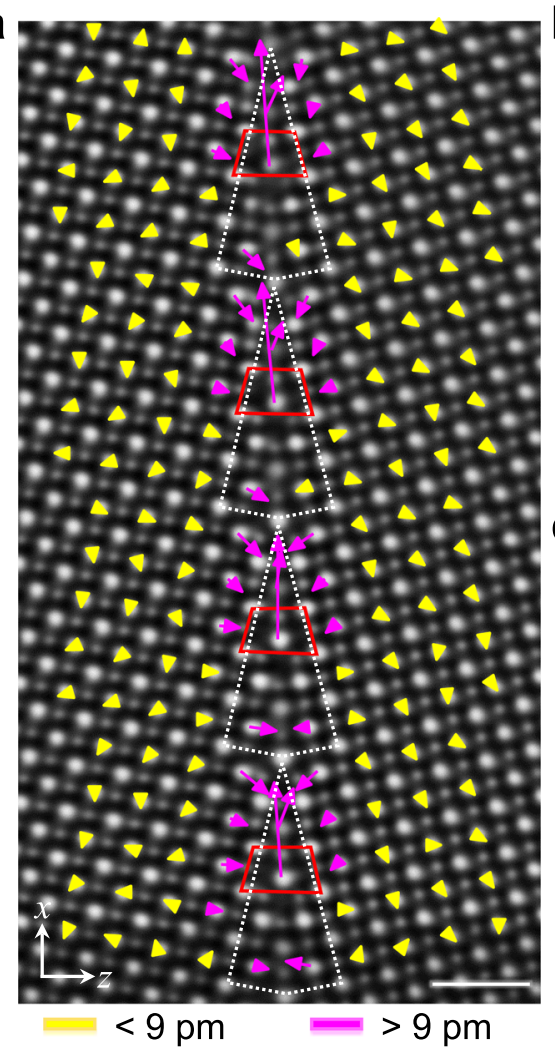

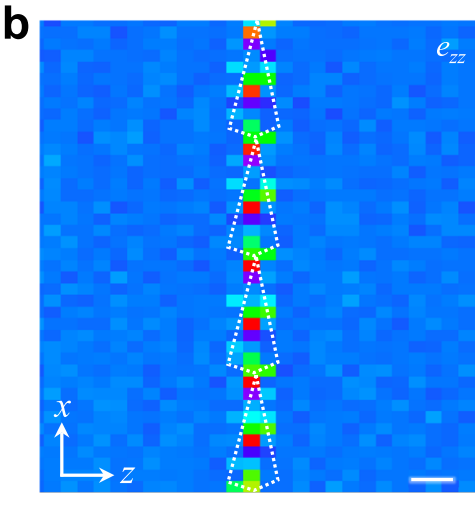
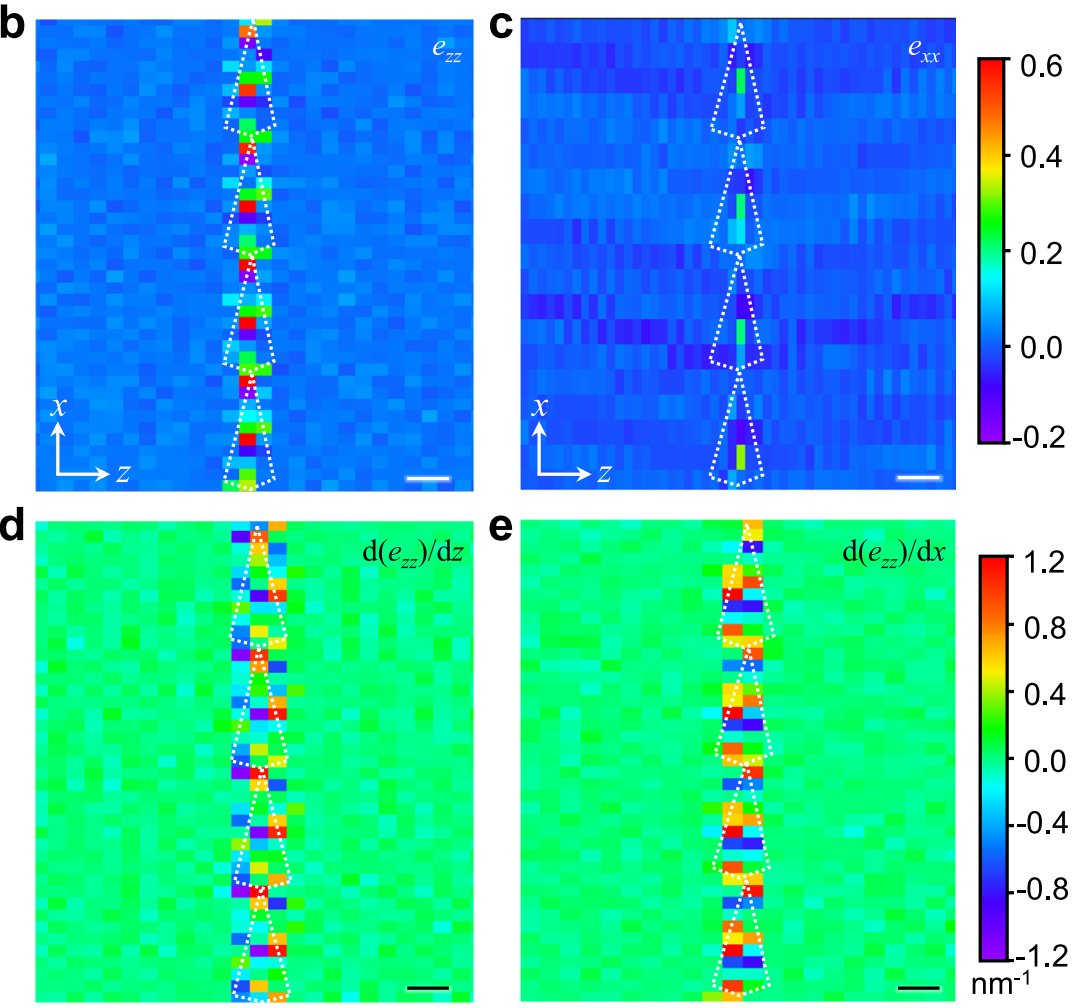

Fig. 3 Atomic-scale displacements and strain gradients in the LAO GB. a Off-center displacement vector map between the La and AlO columns from the iDPC image. The structural units of the GB are highlighted by the white polygons. The arrows represent the magnitude and direction of polar displacements estimated based on the offsets of the AIO column with respect to the center of the surrounding four La columns. Scale bar, $1 \mathrm{~nm}$. b, c Unit cell-scale mapping of $e_{z z}(\mathbf{b})$ and $e_{x x}(\mathbf{c})$ corresponding to the strain perpendicular and parallel to the GB plane, respectively. Scale bar, $1 \mathrm{~nm}$. d, e Strength of the strain gradients from $e_{z z}$. d, e Horizontal $\left(d\left(e_{z z}\right) / d z\right)$ and vertical $\left(d\left(e_{z z}\right) / d x\right)$ strain gradients, respectively. The structural units of the $G B$ are highlighted by the white polygons. Scale bar, $1 \mathrm{~nm}$.

simulations (Supplementary Fig. 5a) shows that electrons have a sharp maximum value at the GB, which can effectively screen the depolarizing fields and stabilize the head-to-head polarization configurations. Moreover, four-dimensional STEM (4D-STEM) measurements (see details in the "Methods" section) in Supplementary Fig. 5b-d further validate the presence of negative charge accumulation at the GB, which certainly plays an important role in determining the electrical activities of the GB in these electroceramics.

Electrical structure of the GB. Therefore, we performed EELS at the oxygen $K$-edge and the aluminum $L$-edge to investigate the electronic structure of the flexoelectric phase in the GB. For reliability and accuracy of the data, we carefully chose clean and uniform regions to perform EELS measurements, as shown in Supplementary Fig. 6 (see details in the "Methods" section). The spectra from the bulk matrix and the GB core are extracted in Fig. $4 \mathrm{~b}, \mathrm{c}$. The oxygen $K$-edge spectra (Fig. $4 \mathrm{~b}$ ) illustrate a similar peak at $536.5 \mathrm{eV}$ in the bulk and GB core, which is related to La $5 d-\mathrm{O} 2 p$ hybridized states ${ }^{33}$, while a feature at approximately $533.5 \mathrm{eV}$ (peak A) only exists in the GB, indicating stronger La-O interactions $s^{34}$. Spectral region $\mathrm{B}$ corresponds to the transition to $\mathrm{O} 2 p$ states hybridized with $\mathrm{Al} 3 p$ states $^{34,35}$. The different fine structure of spectral region $\mathrm{B}$ in the GB demonstrates the modified hybridization between $\mathrm{Al}$ and $\mathrm{O}$ states. A previous study reported that the hybridization between $\mathrm{Ti} 3 d$ and $\mathrm{O} 2 p$ in $\mathrm{PbTiO}_{3}$ and $\mathrm{BaTiO}_{3}$ accounts for their ferroelectricity ${ }^{36}$. Therefore, the stronger La-O hybridization in the GB core is in well agreement with the presence of flexoelectricity. Additionally, the Al $L$-edge spectrum generally exhibits a fingerprint that signifies the change in the $\mathrm{Al}$ coordination symmetry and reflects the unoccupied conduction band minimum $(\mathrm{CBM})^{37-39}$. As shown in Fig. 4c, the decrease in the threshold energy at the $\mathrm{Al} L_{3}$-edge highlighted by the arrow is $\sim 0.6 \mathrm{eV}$, illustrating distorted octahedral symmetry in the GB area ${ }^{40}$, which is also reflected in the difference in the fine structure at the $\mathrm{Al} L_{1}$-edge (Supplementary Fig. $7 a)^{41}$ and the substantial reduction in the $L_{3} / L_{2}$ ratio (depicted in Supplementary Fig. 7b). Furthermore, because the $\mathrm{Al}$ $L_{2,3}$-edges originate from the electron excitations from the $\mathrm{Al} 2 p$ to unoccupied $\mathrm{Al} 3 s$ states, while the $\mathrm{Al} 3 s$ orbital dominates the $\mathrm{CBM}^{39}$, the lower onset energy may also manifest a band gap reduction in the GB, which is confirmed by DFT calculations of the density of states (Fig. 4d) and electronic band structure (Supplementary Fig. 8).

\section{Discussion}

To validate the generality of the flexoelectricity in the GB, we then investigated $\mathrm{SrTiO}_{3}$ (STO) GBs to present the universal structural information associated with adjustable flexoelectricity. As shown in Supplementary Fig. 9, the maximum strain gradient in the $22.6^{\circ}$ STO GB is evaluated as $\sim 1.5 \mathrm{~nm}^{-1}$ for $\mathrm{d}\left(e_{z z}\right) / \mathrm{d} x$, resulting in a cationic atomic shift of up to $60.3 \pm 5.7 \mathrm{pm}$. The strain gradients are more pronounced in the $36.8^{\circ}$ STO GB core $\left(\sim 1.9 \mathrm{~nm}^{-1}\right.$ for $\left.\mathrm{d}\left(e_{z z}\right) / \mathrm{d} x\right)$, inducing giant atomic shifts up to $\sim 119.1 \pm 4.3 \mathrm{pm}$ (Supplementary Fig. 10). Note that such an enhancement of displacements might not only be caused by flexoelectricity but 
a
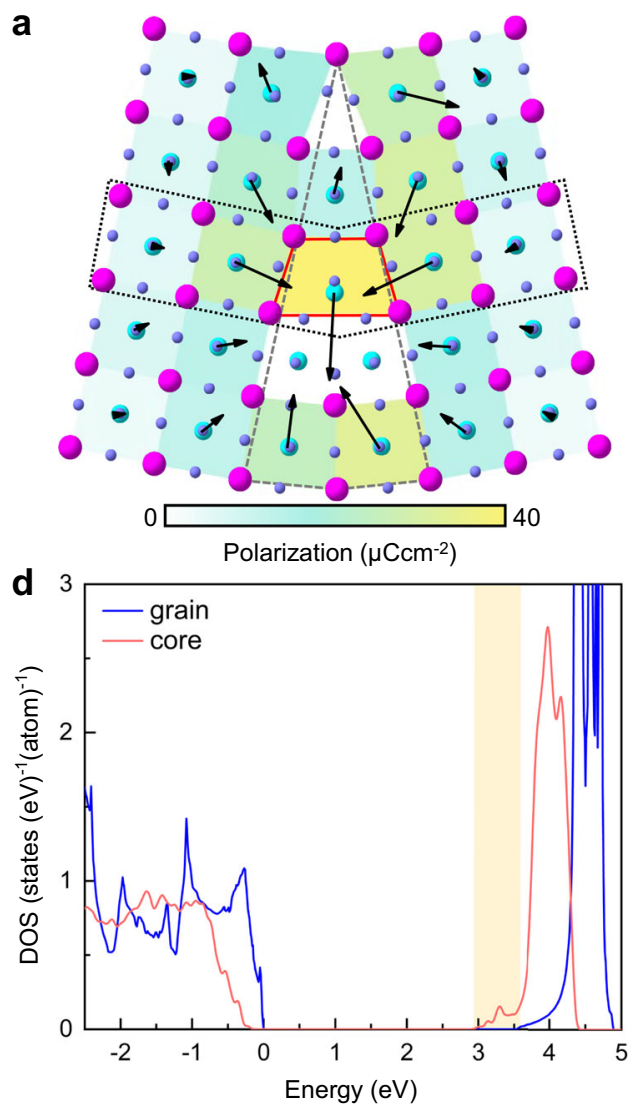

b

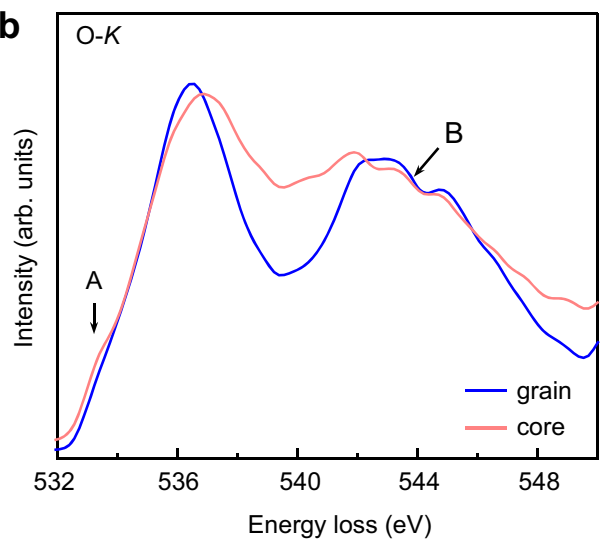

C

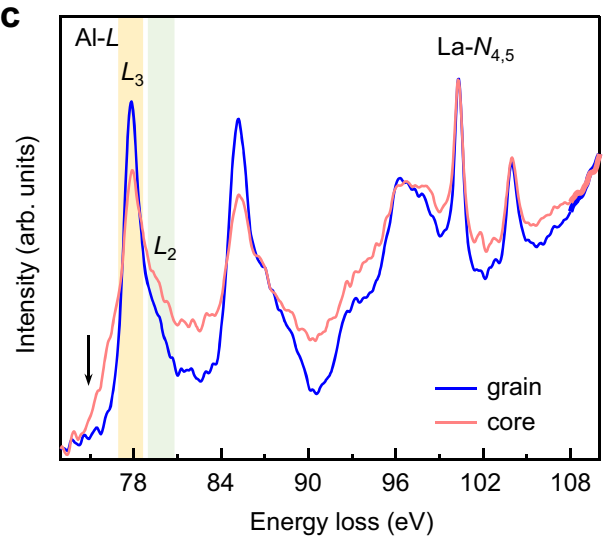

Fig. 4 Polarization and electronic structures of the LAO GB. a Atomic structure and polarization of the GB from DFT calculations. Vectors denote the polarization direction for each unit cell. The strength of polarization is expressed as a color map, ranging from white (weak) to yellow (strong). In the red polyhedron, the displacement between the cations and oxygen atoms in the AlO octahedron is clearly visible with an upward component, accounting for the deviation between the polar vector and displacement vector. $\mathbf{b} O \mathrm{~K}$-edges from the $\mathrm{GB}$ core (red) and grains (blue) indicating stronger hybridization of La-O interactions in the GB. c Al L-edges and La N-edges manifesting different local Al-O configurations in the GB. The corresponding Al $L_{3}$ (yellow) and $L_{2}$ (green)-edges are marked for clarity. $\mathbf{d}$ Calculated density of states of the GB core (red) and grains (blue). The valence band maximum (VBM) energies in the bulk and at the GB are set to zero.

also partly come from the nonstoichiometry which are usually strongly coupled with each other ${ }^{23}$. We also measured the EELS spectra for different STO GBs, and found them very different. For example, the $22.6^{\circ} \mathrm{STO}$ GB shows more decreased crystal field splitting (Supplementary Fig. 11). However, it should also be noted that in addition to the flexoelectricity, the electrical changes in the GB are probably influenced by multiple factors, such as bonding changes, possible local charge changes, and their interactions, rather than only flexoelectricity. On the other hand, the strain gradients in the LAO and STO GB cores measured by experiments (depicted in Fig. 1b and highlighted by red dots), show good consistency with the estimated geometric relation, indicating that a wide range of strain gradients can be obtained by controlling the GB angle. In addition to tunability and universality, GB engineering has advantages in generating flexoelectricity compared with other strategies, such as changing the epitaxial conditions or other defects. For example, although great tensile strains exist in the center of cracks in $\mathrm{STO}^{42}$, symmetric stretching prevents the flexoelectric polarization response due to the small strain gradients. In contrast, the GB restricts the large strain gradients to within a few unit cells, serving to deterministically trigger nanoscale flexoelectricity.

Moreover, the polarized GB core further influences the electrostatic potential and the electronic density in the GB, modulating the electrical activities. Taking LAO as an example, a stronger hybridization state is located in the GB core, favoring and promoting the formation of polar distortion. The decrease in the $\mathrm{Al} L_{3}$ onset energy indicates disordered configuration complexity, which may allow weak $p-d$ hybridized states that are forbidden in the perfect $\mathrm{AlO}_{6}$ octahedral configuration, possibly causing a small number of defective states between the valence band maximum (VBM) and $\mathrm{CBM}^{39,40}$. In addition, around the $\mathrm{GB}$, the flexoelectric distortion may also couple with the rotational distortion (e.g., antiferrodistortive distortion at the $22.6^{\circ}$ STO $\mathrm{GB}^{21}$ ), giving rise to additional effects such as improper ferroelectricity ${ }^{43}$ and the rotostriction effect ${ }^{44}$ and thus influencing the electronic properties of the GB in a more complicated fashion.

Although GBs is well-known to play critical roles in the properties of oxide ceramics, the underlying mechanism is far from clear due to the intricacy. Among all the proposed mechanisms, elemental segregation-induced local nonstoichiometry ${ }^{45,46}$ is usually believed to be the dominant mechanism, which changes the local charge conditions and influences the transport properties ${ }^{47-49}$. In our study, the observed universal nanoscale flexoelectricity at GBs in these oxide ceramics provides a scenario to understand the electrical activities, i.e., the flexoelectric dipole carries "internal" polarization, and then, the bound charge must be screened by the "external" charges or it will not stably exist ${ }^{50}$, inducing charge (electron, oxygen vacancy, hole, etc.) redistribution and thus being expected to likewise influence the electrical activities. Furthermore, we built 
a structure that only has off-stoichiometry without huge strain gradients, showing a much smaller dipole moment $\left(\sim 9 \mu \mathrm{C} \mathrm{cm}^{-2}\right)$ compared with the polarization of the trapezoidal cell in the GB core. Hence, the unique trapezoidal unit cell-induced flexoelectricity has a significant influence on the polar displacements and hence electrical activity. In this sense, the role played by the huge strain gradient-induced flexoelectricity is as important as the commonly believed elemental segregation for GBs.

In summary, we establish a systematic picture of nanoscale flexoelectricity at GBs, in which tunable and huge strain gradients can be achieved at the atomic scale. By the advanced STEM technique and theoretical DFT calculations, we determine the overall atomic structure of the exotic trapezoidal-like configuration in the GB, evaluate the strain gradients, quantify the atomicscale flexoelectricity, reveal the electronic structure, and demonstrate the generality. Due to the huge strain gradients, large offdisplacements of more than a hundred picometers can be achieved in the GB core with a polarized area typically within 3 4-unit cells. The presence of considerable flexoelectricity in the GBs and its universality provide insights into understanding the electrical activities of GBs in electroceramics.

\begin{abstract}
Methods
Fabrication of bicrystals. A LAO bicrystal with a $24^{\circ}$ mistilt GB was fabricated by using thermal diffusion bonding of two LAO single crystals at $1800^{\circ} \mathrm{C}$ for $12 \mathrm{~h}$ in a $\mathrm{N}_{2}$ atmosphere under a uniaxial stress of $2 \mathrm{MPa}$. A STO bicrystal with a $36^{\circ}$ mistilt GB was fabricated based on thermal diffusion bonding of two STO single crystals at $1000{ }^{\circ} \mathrm{C}$ for $10 \mathrm{~h}$ in a $\mathrm{N}_{2}$ atmosphere under a uniaxial stress of $2 \mathrm{MPa}$. The bicrystal was supplied by Hefei Ke Jing Materials Technology Co., LTD.
\end{abstract}

Preparation of TEM samples. The TEM specimens were first thinned by mechanical polishing and then subjected to argon ion milling. The ion milling process was carried out using a PIPS ${ }^{\mathrm{mt}}$ (Model 691, Gatan, Inc.) with an accelerating voltage of $3.5 \mathrm{kV}$ until a hole was observed. Low voltage milling was performed with an accelerating voltage of $0.3 \mathrm{kV}$ to remove the surface amorphous layer and minimize the irradiation-damaged layers.

Electron microscopy characterization and image analysis. HAADF and IDPC images were recorded at $300 \mathrm{kV}$ using an aberration-corrected FEI Titan Themis G2. The convergence semiangle for imaging was $30 \mathrm{mrad}$, and the collection semiangle snap range was 4-21 mrad for iDPC imaging and 39-200 mrad for HAADF imaging. To obtain a sufficient signal-to-noise ratio for quantitative analysis, the iDPC image was acquired at $4096 \times 4096$ pixels, with a dwell time of $500 \mathrm{~ms} \mathrm{pixel}^{-1}$ and a beam current of $20 \mathrm{pA}$ to avoid beam damage.

To obtain quantitative information on the flexoelectricity, the atom positions were determined by simultaneous fitting with two-dimensional Gaussian peaks using a MATLAB code. The magnitude of the strain was defined as the difference in the lattice parameters between the strained and ground states. The strain was calculated based on the $\mathrm{La}(\mathrm{Sr})$ sublattice as strain $=\frac{a-a_{0}}{a_{0}}$, where $a_{0}$ is the lattice parameter of free-standing LAO (STO) and $a$ is the in-plane lattice parameter perpendicular $\left(a_{z}\right)$ or parallel $\left(a_{x}\right)$ to the GB plane. The strain gradient was then obtained by taking the differential of the strains along different directions, given as $\mathrm{d}\left(e_{z z}\right) / \mathrm{d} z=2 *\left(e_{z z}^{n}-e_{z z}^{n-1}\right) /\left(a_{z}^{n}+a_{z}^{n-1}\right)$ and $\left.\mathrm{d}\left(e_{z z}\right) / \mathrm{d} x=\left(e_{z z}^{m}-e_{z z}^{m-1}\right) / a_{x}^{m}\right)$. In Fig. $1 \mathrm{~b}$, we recognize side $c$ as the lattice parameter $\left(a_{0}\right)$ of the free-standing state. GPA was performed using the free FRWRtools plugin for Digital Micrograph (developed by Kotch et al. and available at https://www.physics.hu-berlin.de/en/ sem) based on the original work ${ }^{31}$.

A previous study ${ }^{51}$ suggested that the error in displacement measurement mainly comes from misalignments (e.g., specimen mistilt and residual lens aberration). In our work, we conducted careful alignment to minimize the artifacts. We set the center of the Ronchigram of the GB to the alignment center when acquiring the image, as shown in Supplementary Fig. 2a. The corresponding Ronchigrams of two surrounding grains are also shown in Supplementary Fig. $2 b, c$. Then, the offset between the center of the Ronchigram of surrounding grains and the alignment center indicates the deviation from their on-zone axis orientations (nearly $1.7 \mathrm{mrad}$, corresponding to $0.1^{\circ}$ ). Based on the experimental parameters, we performed a QSTEM software simulation (https:// www.physics.hu-berlin.de/en/sem/software/software_qstem). The results show that such crystal tilts will result in $\sim 1 \mathrm{pm}$ false displacement measurements (Supplementary Fig. 2d). Furthermore, the surrounding grains away from the GB core were regarded as the reference polarization-free structure and analyzed to evaluate the mistilt effect. Then, the displacements in the reference LAO and STO were measured as $4.3 \pm 1.8 \mathrm{pm}$ (Supplementary Fig. 2e) and $4.8 \pm 1.6 \mathrm{pm}$, showing good agreement with the previous iDPC image error level (typically $\sim 5 \mathrm{pm}^{51}$ ). The discrepancy between the simulation and experiment mainly arises because the experimental error comes from not only mistilt noise but also scanning noise and specimen drift in acquiring STEM images ${ }^{30}$. Nevertheless, the error level is typically one order below our flexoelectric displacement ( $\sim 81.4 \mathrm{pm}$ ), proving the data reliability. To further minimize the fluctuation between different locations, the estimated displacements in the GB core were averaged among the periodic structural units to present the key features.

Four-dimensional scanning electron diffraction (4D-STEM) characterizations Scanning diffraction data sets were collected by using a Nion HERMES 200 microscope operated at $60 \mathrm{kV}$. The probe convergence semiangle was $35 \mathrm{mrad}$. The sample thickness was estimated as $\sim 6 \mathrm{~nm}$. All acquired $4 \mathrm{D}$ data sets were processed by custom-written Python code based on the GetDPC package ${ }^{52}$. However, such phase contrast in $4 \mathrm{D}$-STEM mode is very sensitive to the crystal tilt ${ }^{53}$ and thickness variation and limit ${ }^{54}$. Thus, achieving an accurate conclusion regarding the quantitative charge analysis for GBs from 4D-STEM measurements remains challenging due to the presence of small misalignment between two grains, stress relaxation in the ultrathin specimen, and possible thickness differences across the GB.

EELS characterizations and analysis. STEM-EELS spectra were recorded using a Nion HERMES 200 microscope. To reduce beam damage, EELS experiments were performed at $60 \mathrm{kV}$. The probe convergence semiangle was $35 \mathrm{mrad}$, and the collection semiangle was in the range of $24.9 \mathrm{mrad}$. We acquired a single $25 \times 70$ pixel EELS map from a $4.27 \times 16 \mathrm{~nm}$ region containing the GB. The dwell time was $150 \mathrm{~ms} \mathrm{pixel}^{-1}$, and the dispersion was $0.1663 \mathrm{eV} \mathrm{ch}^{-1}$. The data were smoothed by a Gaussian-weighted moving average filter with a window length of 7 channels and a variance of 1 channel. The extracted EELS spectra in Fig. $4 \mathrm{~b}, \mathrm{~d}$ were spatially averaged over the regions parallel to the GB plane (marked by the rectangle in Supplementary Fig. 6b). The EELS background was fitted and subtracted using the power law $I(\triangle E)=A_{0} \cdot \Delta E^{-r}$. The $L_{3} / L_{2}$ ratio calculation was based on the spectral integral intensity ratio of the $77.2-78.8 \mathrm{eV}$ and $79.3-80.9 \mathrm{eV}$ energy windows. The fitting uncertainty was estimated by the standard deviation among rows.

First-principles calculations. Electronic structure calculations were performed using the Vienna $\mathrm{Ab}$ initio Simulation Package (VASP) ${ }^{55,56}$. The projected aug mented wave (PAW $)^{57,58}$ potential was used to address with the interactions between ions and electrons. The exchange-correlation potential was treated by the Perdew-Burke-Ernzerhof ${ }^{59}$ functional. A plane-wave cutoff of $600 \mathrm{eV}$ was used. For the bulk rhombohedral structure, the experimental lattice constants $(a=b=$ $c=5.360 \AA, \alpha=\beta=\gamma=60.011^{\circ}$ ) were used. Then, the bulk pseudocubic structure was used as a block unit to build the GB structure ${ }^{60}$. Atomic positions were relaxed until the maximum force was less than $0.01 \mathrm{eV} \AA^{-1}$. Band structures were obtained at the DFT level. For the GB, we found that the charge-neutral system is gapless. Thus, to make the system have a finite gap, extra four electrons were added to the cell. Born effective charges were computed using density functional perturbation theory ${ }^{61-63}$.

The polarization calculation was based on the equation of spontaneous polarization $P_{s}=\frac{1}{V} \sum \delta_{i} Z_{i}{ }^{64}$, where $V$ is the volume of the unit cell, $\delta$ is the displacement of atom, $i$ from its centrosymmetric position and $Z$ is the Born effective charge of atom $i . \delta$ was evaluated from the displacements of each column with respect to the corresponding position in the polarization-free bulk crystal. The charge density was calculated within the DFT in the VASP package.

\section{Data availability}

The authors declare that all relevant data are included in the paper and Supplementary Information files and are available from the corresponding author upon reasonable request.

\section{Code availability}

Additional data including the codes are available from the corresponding author upon reasonable request.

Received: 5 August 2021; Accepted: 17 December 2021; Published online: 11 January 2022

\section{References}

1. Tagantsev, A. K. Piezoelectricity and flexoelectricity in crystalline dielectrics. Phys. Rev. B 34, 5883-5889 (1986).

2. Nguyen, T. D., Mao, S., Yeh, Y. W., Purohit, P. K. \& McAlpine, M. C. Nanoscale flexoelectricity. Adv. Mater. 25, 946-974 (2013).

3. Zubko, P., Catalan, G., Buckley, A., Welche, P. R. \& Scott, J. F. Strain-gradientinduced polarization in $\mathrm{SrTiO}_{3}$ single crystals. Phys. Rev. Lett. 99, 167601 (2007). 
4. Majdoub, M. S., Sharma, P. \& Cagin, T. Enhanced size-dependent piezoelectricity and elasticity in nanostructures due to the flexoelectric effect. Phys. Rev. B 77, 125424 (2008).

5. Yudin, P. V. \& Tagantsev, A. K. Fundamentals of flexoelectricity in solids. Nanotechnology 24, 432001 (2013).

6. Tang, Y. L., Zhu, Y. L., Liu, Y., Wang, Y. J. \& Ma, X. L. Giant linear strain gradient with extremely low elastic energy in a perovskite nanostructure array. Nat. Commun. 8, 15994 (2017).

7. Tang, Y. L. et al. Ferroelectrics. Observation of a periodic array of flux-closure quadrants in strained ferroelectric $\mathrm{PbTiO}_{3}$ films. Science 348, 547-551 (2015).

8. Zou, M. J. et al. Flexoelectricity-induced retention failure in ferroelectric films. Acta Mater. 196, 61-68 (2020)

9. Tang, Y. et al. Periodic polarization waves in a strained, highly polar ultrathin $\mathrm{SrTiO}_{3}$. Nano Lett. 21, 6274-6281 (2021)

10. Park, S. M. et al. Selective control of multiple ferroelectric switching pathways using a trailing flexoelectric field. Nat. Nanotechnol. 13, 366-370 (2018).

11. Catalan, G. et al. Flexoelectric rotation of polarization in ferroelectric thin films. Nat. Mater. 10, 963-967 (2011).

12. Chandratre, S. \& Sharma, P. Coaxing graphene to be piezoelectric. Appl. Phys. Lett. 100, 023114 (2012).

13. Sharma, N. D., Maranganti, R. \& Sharma, P. On the possibility of piezoelectric nanocomposites without using piezoelectric materials. J. Mech. Phys. Solids 55, 2328-2350 (2007).

14. Liu, X. et al. Anomalous photovoltaic effect in centrosymmetric ferroelastic BiVO4. Adv. Mater. 30, 1801619 (2018).

15. Eliseev, E. A., Morozovska, A. N., Glinchuk, M. D. \& Blinc, R. Spontaneous flexoelectric/flexomagnetic effect in nanoferroics. Phys. Rev. B 79, 165433 (2009).

16. Carpenter, M. A., Salje, E. K. H. \& Howard, C. J. Magnetoelastic coupling and multiferroic ferroelastic/magnetic phase transitions in the perovskite $\mathrm{KMnF}_{3}$. Phys. Rev. B 85, 224430 (2012)

17. Meirzadeh, E. et al. Surface pyroelectricity in cubic $\mathrm{SrTiO}_{3}$. Adv. Mater. 31, e1904733 (2019)

18. Shibata, N. et al. Nonstoichiometric dislocation cores in $\alpha$-alumina. Science 316, 82-85 (2007).

19. $\mathrm{Li}, \mathrm{X}$. et al. Atomic origin of spin-valve magnetoresistance at the $\mathrm{SrRuO}_{3}$ grain boundary. Natl Sci. Rev. 7, 755-762 (2020)

20. Meiners, T., Frolov, T., Rudd, R. E., Dehm, G. \& Liebscher, C. H. Observations of grain-boundary phase transformations in an elemental metal. Nature 579, 375-378 (2020).

21. Han, B. et al. Two-dimensional room-temperature giant antiferrodistortive $\mathrm{SrTiO}_{3}$ at a grain boundary. Phys. Rev. Lett. 126, 225702 (2021).

22. Marincel, D. M. et al. Influence of a single grain boundary on domain wall motion in ferroelectrics. Adv. Funct. Mater. 24, 1409-1417 (2014).

23. Gao, P. et al. Atomic-scale measurement of flexoelectric polarization at $\mathrm{SrTiO}_{3}$ dislocations. Phys. Rev. Lett. 120, 267601 (2018).

24. Eliseev, E. A., Morozovska, A. N., Glinchuk, M. D. \& Kalinin, S. V. Lost surface waves in nonpiezoelectric solids. Phys. Rev. B 96, 045411 (2017).

25. Wang, B., Gu, Y., Zhang, S. \& Chen, L.-Q. Flexoelectricity in solids: progress, challenges, and perspectives. Prog. Mater. Sci. 106, 100570 (2019).

26. Salje, E. K. et al. Direct observation of polar tweed in $\mathrm{LaAlO}_{3}$. Sci. Rep. 6 , 27193 (2016).

27. Feng, B. et al. Atomically ordered solute segregation behaviour in an oxide grain boundary. Nat. Commun. 7, 11079 (2016).

28. Wang, Z., Saito, M., Mckenna, K. P. \& Ikuhara, Y. Polymorphism of dislocation core structures at the atomic scale. Nat. Commun. 5, 3239 (2014).

29. Lazić, I., Bosch, E. G. \& Lazar, S. Phase contrast STEM for thin samples: integrated differential phase contrast. Ultramicroscopy 160, 265-280 (2016)

30. Gao, P. et al. Picometer-scale atom position analysis in annular bright-field STEM imaging. Ultramicroscopy 184, 177-187 (2018)

31. Hÿtch, M. J., Snoeck, E. \& Kilaas, R. Quantitative measurement of displacement and strain fields from HREM micrographs. Ultramicroscopy $\mathbf{7 4}$, 131-146 (1998).

32. Wang, Y. J., Tang, Y. L., Zhu, Y. L., Feng, Y. P. \& Ma, X. L. Converse flexoelectricity around ferroelectric domain walls. Acta Mater. 191, 158-165 (2020).

33. Ristic, Z. et al. Nanoscale modulation of the density of states at the conducting interface between $\mathrm{LaAlO}_{3}$ and $\mathrm{SrTiO}_{3}$ band insulators. EPL 93, 17004 (2011).

34. Gauquelin, N. et al. Atomically resolved EELS mapping of the interfacial structure of epitaxially strained $\mathrm{LaNiO}_{3} / \mathrm{LaAlO}_{3}$ superlattices. Phys. Rev. B 90, 195140 (2014)

35. Palina, N. et al. Electronic defect states at the $\mathrm{LaAlO}_{3} / \mathrm{SrTiO}_{3}$ heterointerface revealed by $\mathrm{O}$ K-edge X-ray absorption spectroscopy. Phys. Chem. Chem. Phys. 18, 13844-13851 (2016).

36. Cohen, R. E. Origin of ferroelectricity in perovskite oxides. Nature 358 136-138 (1992).

37. Weigel, C., Calas, G., Cormier, L., Galoisy, L. \& Henderson, G. S. Highresolution $\mathrm{Al} \mathrm{L2,3-edge} \mathrm{X-ray} \mathrm{absorption} \mathrm{near} \mathrm{edge} \mathrm{structure} \mathrm{spectra} \mathrm{of} \mathrm{Al}$ containing crystals and glasses: coordination number and bonding information from edge components. J. Phys. Condens. Mat. 20, 135219 (2008)

38. Bokhoven, J. A. V., T Nabil, H. S., Ramake, D. E. \& Koningsberger, D. C. Interpretation of the $\mathrm{Al} \mathrm{K}$ - and LII/III-edges of aluminium oxides: differences between tetrahedral and octahedral $\mathrm{Al}$ explained by different local symmetries. J. Phys. Condens. Mat. 13, 10247-10260 (2001).

39. Filatova, E. O. \& Konashuk, A. S. Interpretation of the changing the band gap of $\mathrm{Al}_{2} \mathrm{O}_{3}$ depending on its crystalline form: connection with different local symmetries. J. Phys. Chem. C 119, 20755-20761 (2015).

40. Wei, J. et al. Direct measurement of electronic band structures at oxide grain boundaries. Nano Lett. 20, 2530-2536 (2020).

41. Nufera, S., Gemminga, T., Elsässera, C., Köstlmeier, S. \& Rühle, M. Core-hole effect in the ELNES of $\mathrm{a}-\mathrm{Al}_{2} \mathrm{O}_{3}$ experiment and theory. Ultramicroscopy 86, 339-342 (2001).

42. Wang, H. et al. Direct observation of huge flexoelectric polarization around crack tips. Nano Lett. 20, 88-94 (2020).

43. Bousquet, E. et al. Improper ferroelectricity in perovskite oxide artificial superlattices. Nature 452, 732-736 (2008).

44. Schiaffino, A. \& Stengel, M. Macroscopic polarization from antiferrodistortive cycloids in ferroelastic $\mathrm{SrTiO}_{3}$. Phys. Rev. Lett. 119, 137601 (2017).

45. Chiang, Y.-M. \& Takag, T. Grain-boundary chemistry of barium titanate and strontium titanate: I, High-temperature equilibrium space charge. J. Am. Ceram. Soc. 73, 3278 (1990).

46. Waser, R. Electronic properties of grain boundaries in $\mathrm{SrTiO}_{3}$ and $\mathrm{BaTiO}_{3}$, ceramics. Solid State Ion. 75, 89 (1995).

47. Sato, Y. et al. Role of $\mathrm{Pr}$ segregation in acceptor-state formation at $\mathrm{ZnO}$ grain boundaries. Phys. Rev. Lett. 97, 106802 (2006)

48. Klie, R. F. et al. Enhanced current transport at grain boundaries in high-Tc superconductors. Nature 435, 475-478 (2005).

49. Guo, X. \& Waser, R. Electrical properties of the grain boundaries of oxygen ion conductors: acceptor-doped zirconia and ceria. Prog. Mater. Sci. 51, 151-210 (2006).

50. Kalinin, S. V., Kim, Y., Fong, D. D. \& Morozovska, A. N. Surface-screening mechanisms in ferroelectric thin films and their effect on polarization dynamics and domain structures. Rep. Prog. Phys. 81, 036502 (2018).

51. Sun, Y. et al. Subunit cell-level measurement of polarization in an individual polar vortex. Sci. Adv. 5, eaav4355 (2019).

52. Hachtel, J. A., Idrobo, J. C. \& Chi, M. Sub-Ångstrom electric field measurements on a universal detector in a scanning transmission electron microscope. Adv. Struct. Chem. Imaging 4, 10 (2018).

53. Shao, Y.-T. et al. Decoupling polarization, crystal tilt and symmetry in epitaxially-strained ferroelectric thin films using 4D-STEM. Microsc. Microanal. 25, 1938-1939 (2019).

54. Müller-Caspary, K. et al. Measurement of atomic electric fields and charge densities from average momentum transfers using scanning transmission electron microscopy. Ultramicroscopy 178, 62-80 (2017).

55. Kresse, G. \& Furthmüller, J. Efficiency of ab-initio total energy calculations for metals and semiconductors using a plane-wave basis set. Comput. Mater. Sci. 6, 15-50 (1996)

56. Kresse, G. \& Furthmüller, J. Efficient iterative schemes for ab initio totalenergy calculations using a plane-wave basis set. Phys. Rev. B 54, 11169-11186 (1996).

57. Kresse, G. \& Joubert, D. From ultrasoft pseudopotentials to the projector augmented-wave method. Phys. Rev. B 59, 1758-1775 (1999).

58. Blochl, P. E. Projector augmented-wave method. Phys. Rev. B 50, 17953-17979 (1994).

59. Perdew, J. P., Burke, K. \& Ernzerhof, M. Generalized gradient approximation made simple. Phys. Rev. Lett. 77, 3865-3868 (1996).

60. Cheng, J., Luo, J. \& Yang, K. Aimsgb: an algorithm and open-source python library to generate periodic grain boundary structures. Comput. Mater. Sci. 155, 92-103 (2018).

61. Gajdoš, M., Hummer, K., Kresse, G., Furthmüller, J. \& Bechstedt, F. Linear optical properties in the projector-augmented wave methodology. Phys. Rev. B 73, 045112 (2006)

62. Gonze, X. \& Lee, C. Dynamical matrices, Born effective charges, dielectric permittivity tensors, and interatomic force constants from density-functional perturbation theory. Phys. Rev. B 55, 10355 (1997).

63. Baroni, S., de Gironcoli, S., Dal Corso, A. \& Giannozzi, P. Phonons and related crystal properties from density-functional perturbation theory. Rev. Mod. Phys. 73, 515 (2001).

64. Zhong, W., King-Smith, R. D. \& Vanderbilt, D. Giant LO-TO splittings in perovskite ferroelectrics. Phys. Rev. Lett. 72, 3618-3621 (1994).

\section{Acknowledgements}

This work was supported by the National Natural Science Foundation of China (52125307 (P.G.), 11974023 (P.G.), 11774003 (X.-Z.L.), 11934003 (X.-Z.L.)), “2011 Program” Peking-Tsinghua-IOP Collaborative Innovation Center of Quantum Matter, and Strategic Priority Research Program of the Chinese Academy of Sciences Grant No. 
XDB33010400. We acknowledge the Electron Microscopy Laboratory of Peking University for the use of electron microscopes. We are grateful for the computational resources provided by the High-Performance Computing Platform of Peking University.

\section{Author contributions}

P.G. conceived and supervised the project. M.W. performed the HAADF and iDPC image and image analysis assisted by K.Q., Y.W.S., and R.X.Z. under the direction of P.G. X.W.Z performed DFT calculations under the direction of X.-Z.L. M.W implemented the EELS measurements and data analysis assisted by B.H. and K.H.L. M.W implemented the 4D-STEM measurements and the data analysis is dealt with X.Y.G. X.M.L. conducted the EDS experiments with the guidance of X.D.B. All the authors contributed to the analysis of the results.

\section{Competing interests}

The authors declare no competing interests.

\section{Additional information}

Supplementary information The online version contains supplementary material available at https://doi.org/10.1038/s41467-021-27906-0.

Correspondence and requests for materials should be addressed to Xin-Zheng Li or Peng Gao.
Peer review information Nature Communications thanks the other anonymous reviewer(s) for their contribution to the peer review of this work.

Reprints and permission information is available at http://www.nature.com/reprints

Publisher's note Springer Nature remains neutral with regard to jurisdictional claims in published maps and institutional affiliations.

\section{(c) (i)}

Open Access This article is licensed under a Creative Commons Attribution 4.0 International License, which permits use, sharing, adaptation, distribution and reproduction in any medium or format, as long as you give appropriate credit to the original author(s) and the source, provide a link to the Creative Commons license, and indicate if changes were made. The images or other third party material in this article are included in the article's Creative Commons license, unless indicated otherwise in a credit line to the material. If material is not included in the article's Creative Commons license and your intended use is not permitted by statutory regulation or exceeds the permitted use, you will need to obtain permission directly from the copyright holder. To view a copy of this license, visit http://creativecommons.org/ licenses/by/4.0/.

(C) The Author(s) 2022 